\title{
Randomized clinical controlled trial on the effectiveness of conventional and orthodontic manual toothbrushes
}

\section{Lisiane Krieger Gomes ${ }^{(a)}$ Carlos Frederico Sarmento ${ }^{(b)}$ Flávio Roberto Guerra Seabra(b) Patrícia Bittencourt Dutra dos Santos ${ }^{(c)}$ \\ Fábio Henrique de Sa Leitão Pinheiro(d) $^{(d)}$}

\begin{abstract}
The objective of this study was to compare the effectiveness of two manual toothbrushes (conventional and orthodontic). The following clinical parameters were used: VPI (visible plaque index) and GBI (gingival bleeding index). Patients, 64 total (30 males and 34 females), in the permanent dentition, with a mean age of 17.8 years, were randomly selected from a practice specializing in orthodontics. Each participant received audio-visual instructions on oral hygiene as well as a kit of materials containing two manual toothbrushes (orthodontic and conventional). Each toothbrush was randomly allocated to one side of the mouth (splitmouth design) and used for a period of approximately 4 weeks. The VPI and GBI were measured by a single calibrated examiner before $\left(\mathrm{T}_{0}\right)$ and after $\left(\mathrm{T}_{1}\right)$ the implementation of interventions. The Mann-Whitney test was used to compare the VPI values between the groups, and the Student $\mathrm{t}$-test for independent samples was used to compare GBI values. The level of significance was set at $5 \%$. No statistically significant difference was observed between the groups at $\mathrm{T}_{0}$ for both VPI and GBI, and at $\mathrm{T}_{1}$ for the GBI. The manual orthodontic toothbrush produced a statistically lower VPI $(\mathrm{P} \leq 0.05)$ at $\mathrm{T}_{1}$, but this did not seem to be of clinical importance.
\end{abstract}

Descriptors: Orthodontics; Biofilms; Oral Hygiene.

\section{Introduction}

Preventing dental demineralization requires, among other things, frequent removal of the biofilm that accumulates on the surface of teeth. Simple as it may sound, brushing teeth regularly is a task frequently neglected by some individuals, not to mention the occasions when brushing is not done appropriately.

In orthodontics, this problem takes on bigger dimensions as those wearing fixed orthodontic appliances are more susceptible to the accumulation of biofilm than the rest of the population. For these patients, the industry has manufactured a series of hygiene aids ranging from easy-to-use dental floss to orthodontic and interproximal toothbrushes ${ }^{1}$ as well as electric ${ }^{2,3}$ and ultrasonic toothbrushes. ${ }^{3-5}$

There has been quite substantial interest in testing the effectiveness of powered hygiene devices for orthodontic patients. Although part of the positive results must be attributed to the psychological impact of using a relatively sophisticated device, some studies have reported a reduction in 
gingival bleeding index (GBI), ${ }^{2}$ visible plaque index (VPI), and in the number of Streptococcus mutans in patients using such devices. ${ }^{6}$

In the midst of the allure of high-tech toothbrushes, studies on the effectiveness of orthodontic manual toothbrushes have had mixed results. A systematic PubMed search of the literature prior to June 17, 2010, combining the descriptors "orthod" and "toothbrush", produced a total of 68 publications. Among the ones written in English, only five had been designed to compare the performance of conventional and orthodontic manual toothbrushes. None of these studies were split-mouth randomized controlled trials, and two of them had relatively small sample sizes (between 10 and 14 patients). In addition, the comparisons were based on an intraindividual experimental design, thus requiring even larger samples.

Of the five selected studies, only one ${ }^{7}$ observed improved outcomes with the orthodontic manual toothbrush. Considering that this study was published in the 1980's, and the fact that orthodontic toothbrushes have gone through advancements in bristle design and material, there appeared to be a need for updated, evidence-based studies using more stringent methods. Manual orthodontic toothbrushes are still recommended by practitioners all over the world, primarily because of active marketing by the industry. Unbiased well-conducted trials are necessary to assess the effectiveness, and even the risks, of common brands of orthodontic toothbrushes during orthodontic treatment.

This split-mouth randomized clinical controlled trial compared the effectiveness of an orthodontic manual toothbrush (Orthodontic, Oral-B) in relation to a conventional homologous design (Sensitive, Oral-B). This study tested the null hypothesis ( $\mathrm{H} 0)$ that there would be no statistically significant reduction in VPI or GBI with either type of intervention after a follow-up time of 1 month.

\section{Methodology}

This split-mouth, parallel, randomized clinical trial was approved by the research ethical board at Potiguar University according to approval protocol number 297/2009. This study conformed to 2001
CONSORT statement requirements.

Patient samples were obtained from a local practice specialized in orthodontic training for postgraduate students. A total of 720 orthodontic records were available for the study. Because 430 patients were already wearing retainers, they were excluded. Of the 290 remaining records, 64 were randomly selected for this study.

The inclusion criteria were permanent dentition, no more than $4 \mathrm{~mm}$ anterior crowding, fixed orthodontic (multibracket) appliances until the $2^{\text {nd }}$ premolars or beyond, maximum age of 35 years, no remarkable periodontal problems, same type of orthodontic mechanics (modified Tweed-Merrifield technique), and same bracket type $(.022 \times .028 \mathrm{slot}$, Roth prescription, Morelli, Sorocaba, Brazil), no enamel defects or restorations involving the buccal surface, no prostheses and good general health. All patients were non-smoking and had not received dental prophylaxis or used oral mouthwashes in the previous 4 weeks. Those being treated with asymmetric extractions were excluded. After initially selecting 56 patients (30 females, 26 males) who fulfilled all of the aforementioned criteria, 8 patients were still needed to comply with the sample size calculation of 64 subjects, based on the statistical parameters (minimal difference between plaque index mean values of 0.25 , standard deviation of 0.32 , significance level of $5 \%$, and power of $80 \%$ ) presented in a previous study. ${ }^{2}$ For this reason, two additional females and six male orthodontic patients, who were eligible to participate, were retrieved from the original list of patients at random.

Students treating the selected patients were provided only with the following written and verbal recommendations:

1. not to perform dental prophylaxis,

2. not to install ancillary mechanics that could interfere with oral hygiene,

3. not to prescribe any type of mechanical or chemical oral hygiene methods,

4. not to discuss any matter pertaining to the study, and

5. not to use any type of bracket ligature other than the conventional elastic ligatures available in the practice. 
Both the students and their clinical mentors were not informed of the objectives of the study.

Patients were requested to fill out a questionnaire to confirm the inclusion criteria. Following this, an intraoral examination was carried out in a dental setting to confirm that any excludible conditions had not been overlooked. When judging patient eligible for the study, both the VPI developed by Silness and Löe, ${ }^{8}$ and the GBI developed by Löe and Silness ${ }^{9}$ were recorded by a single calibrated examiner on a datasheet especially designed to secure data concealment. These were considered to be the baseline $\left(\mathrm{T}_{0}\right)$ values.

All selected patients then received an oral hygiene kit containing the following products:

- dental floss (Essential floss, Oral-B),

- non-alcoholic $0.05 \%$ sodium fluoride mouthwash (Pro-Saude, Oral-B, Iowa City, USA),

- toothpaste and

- two types of Oral-B manual toothbrushes:

- Oral-B Orthodontic and

- Oral-B Sensitive (Pro-Saude, Oral-B, Iowa City, USA).

Both toothbrushes had virtually the same dimensions and design, differing only in regard to the height of the central bristles, which were much shorter in the orthodontic type.

Patients were then provided with written (Figure

\begin{tabular}{c|l}
\hline Items & \multicolumn{1}{c}{ Instructions } \\
\hline Toothpaste & $\begin{array}{l}\text { Request a new tube when you realize that it is almost empty. } \\
\text { Use a pea-size blob only. Do not swallow! }\end{array}$ \\
\hline Dental floss & $\begin{array}{l}\text { Thread the floss above the archwire for your upper teeth and } \\
\text { below it for your lower teeth. Work it past the contact point } \\
\text { where the two teeth touch. Curve the floss around each tooth } \\
\text { in a "C" shape, and gently move it up and down the side of } \\
\text { each tooth, including under the gum line. Unroll a new section } \\
\text { of floss as you move from tooth to tooth. Do this once a day } \\
\text { before going to bed. }\end{array}$ \\
\hline Fluoride- & $\begin{array}{l}\text { Vigorously swirl the mouthwash around your mouth for one } \\
\text { minute after brushing your teeth, and immediately before }\end{array}$ \\
mouthwash & $\begin{array}{l}\text { going to bed. Do not swallow it! Do not rinse it from your } \\
\text { mouth with water. Spit it out as much as you can. }\end{array}$ \\
\hline Right side & $\begin{array}{l}\text { Remember to use it in the right side of your mouth only. } \\
\text { When brushing the outside surfaces of your teeth, try to move } \\
\text { the brush in small circles, a few teeth at a time. Wiggle it from } \\
\text { the gum line toward the chewing surfaces in a very gently } \\
\text { manner. Pay particular attention to the area between the } \\
\text { braces and below or above the gums. They tend to } \\
\text { accumulate plaque. Spend about 10 seconds for each tooth. } \\
\text { You should brush your teeth at least twice a day (in the } \\
\text { morning and before going to bed). }\end{array}$ \\
\hline Left side & $\begin{array}{l}\text { Remember to use it in the left side of your mouth only. Follow } \\
\text { the same instructions as for the right side. }\end{array}$ \\
\hline
\end{tabular}

Figure 1 - Written oral hygiene instructions provided to participating patients.
1) and verbal instructions regarding oral hygiene during orthodontic treatment. To reinforce the oral hygiene instructions, a 10-minute video containing the same information was presented.

A research collaborator who was not going to take part in the subsequent stages of the study was assigned to allocate each toothbrush to each side of the mouth in each patient. Because all patients were right-handed, toothbrush distribution to either side of the mouth had to be determined randomly. Each gender group of 32 patients was dichotomized into two subgroups of 16 patients each:

- Group 1: orthodontic toothbrush assigned to the right side of the mouth + conventional toothbrush assigned to the left side of the mouth, and

- Group 2: the other way around.

Patients were instructed on which side to use each toothbrush, and on the boundaries pertaining to each of them. The handle of each toothbrush was marked with "L" for left and " $R$ " for right side. After providing all instructions, each patient was then booked for a follow-up examination one month later. For each individual, this time varied slightly (1-3 days) depending on the scheduling of their orthodontic review appointment. The mean time from $\mathrm{T}_{0}$ to follow-up $\left(\mathrm{T}_{1}\right)$ was 27.86 days. The mean age at the beginning of the study was 17.8 years $(16.6$ years for males, 18.9 years for females).

During the $T_{1}$ appointment, patients were requested to demonstrate on a study model how they were using both toothbrushes. Hygiene-related information to check compliance with the written instructions was also requested. All patients reported brushing each side of the mouth with the correct toothbrush. Where the written instructions had not been completely followed, this was observed to occur only occasionally within the considered time interval. A single calibrated examiner then assessed each patient's $T_{1}$ values for VPI and GBI, and entered them into the datasheet. The design did not include the collection of any information on enamel damage. Figure 2 is a flowchart of the sample selection process and sequence of examinations.

Baseline $\left(\mathrm{T}_{0}\right)$ and follow-up $\left(\mathrm{T}_{1}\right)$ data were stored in SPSS (Statistical Package for the Social Sciences, 
version 9), and the datasets for the two study groups were subjected to statistical analysis. The Kolmogorov-Smirnov test revealed a normal distribution for the GBI data but not for the VPI data. For this reason, GBI and VPI intergroup comparisons were assessed by the non-paired Student t-test and the Mann-Whitney test, respectively. For all tests, the level of significance was set at $5 \%$.

\section{Results}

Patients, 30 male and 34 female, mean age 17.8 years, participated in the study. The descriptive statistics can be found in Table 1 for both VPI and GBI ( $\mathrm{T}_{0}$ and $\mathrm{T}_{1}$ data).

When comparing the two groups (conventional and orthodontic toothbrushes), no statistically significant difference $(\mathrm{P} \leq 0.05)$ was observed for VPI $\left(\mathrm{T}_{0}\right)$, GBI $\left(\mathrm{T}_{0}\right)$ and GBI $\left(\mathrm{T}_{1}\right)$ (Table 2$)$. There was, however, a statistically significant improvement in VPI with the orthodontic toothbrush when comparing $\mathrm{T}_{1}$ values ( $\mathrm{P}=0.002$; Table 2 ).

\section{Discussion}

Most manufacturers of dental care products have developed toothbrushes specifically designed for patients with braces. The manual orthodontic toothbrush is one of several hygiene devices that orthodontists recommend to their patients. ${ }^{10}$ Therefore, it is of interest if this product lives up to its claims in terms of being advantageous in relation to the conventional manual toothbrush. Our research group decided to compare Oral-B products because this manufacturer supplies the market with two manual toothbrushes that are very much alike, differing only in terms of height of the central bristles, which is the main characteristic for the orthodontic toothbrush.

Prior to this study only five studies specifically addressed the effects of manual orthodontic toothbrushes. ${ }^{7,11-13}$ None of them utilized a split-mouth design to control for confounding factors pertaining to each individual, such as age, lifestyle, diet changes, hormones and systemic factors. The present study also demonstrated the absence of statistically significant differences between VPI and GBI values at $\mathrm{T}_{0}$, which is a reflection of the homogene-

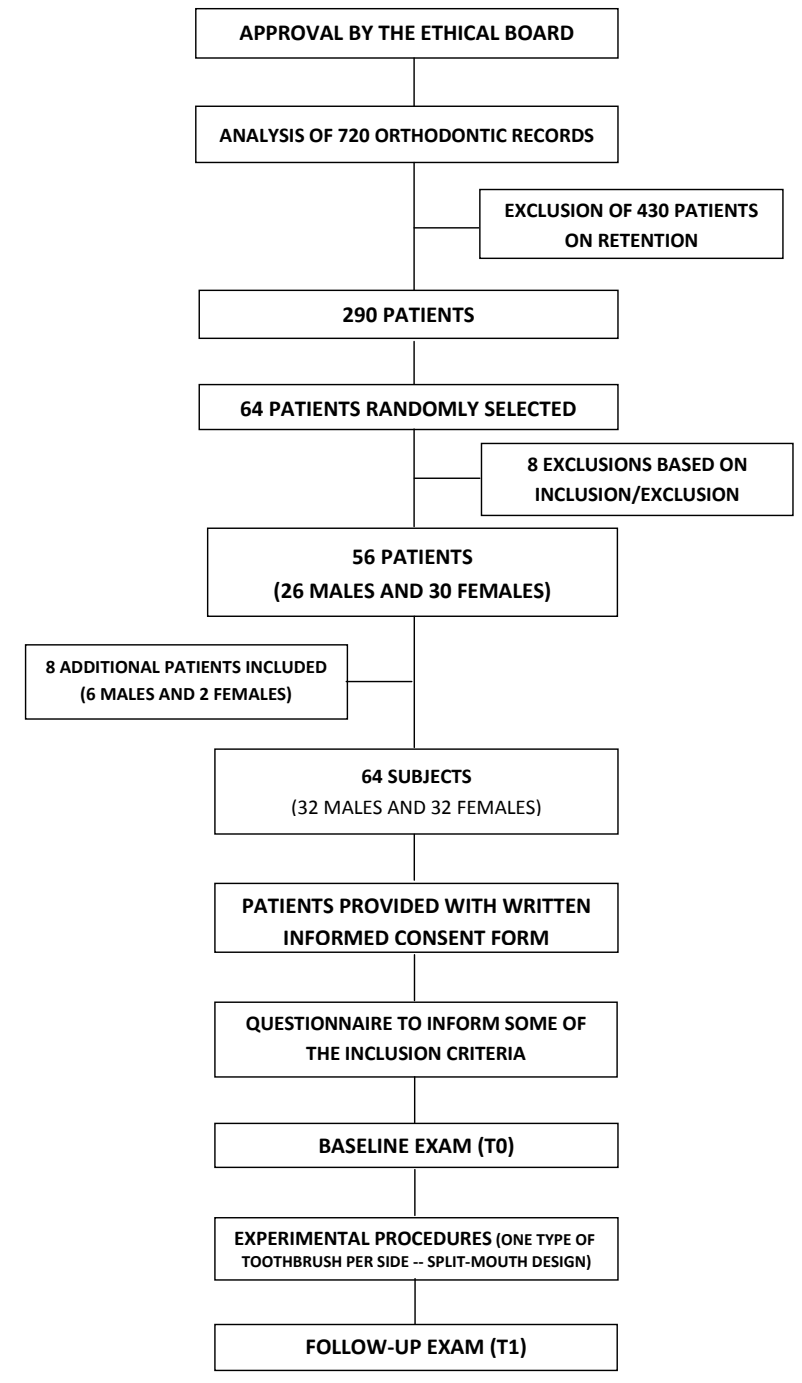

Figure 2 - Flowchart of the sample selection process and sequence of examinations.

ity of the sample (Table 2). In other words, the patients of both groups (orthodontic toothbrush and conventional toothbrush) were comparable in terms of both VPI and GBI during the trial.

The use of Silness and Löe's VPI ${ }^{8}$ instead of Quigley and Hein's ${ }^{14}$ plaque index (as modified by Turesky, Gilmore and Glickman $)^{15}$ was due to the requirement for plaque disclosure in the latter, which implies the need for a prophylaxis to remove the disclosing agent or the substitution of stained elastic ligatures. As the majority of our patients were examined after their periodic review appointment, plaque disclosure could create a certain level of patient discontentment since their teeth and ligatures 
Table 1 - Descriptive statistics for VPI and GBI baseline and follow-up data.

\begin{tabular}{c|c|c|c|c|c|c|c|c}
\hline & $\begin{array}{c}\mathrm{VPI}\left(\mathrm{T}_{0}\right) \\
\mathrm{CT}\end{array}$ & $\begin{array}{c}\mathrm{VPI}\left(\mathrm{T}_{0}\right) \\
\mathrm{OT}\end{array}$ & $\begin{array}{c}\mathrm{VPI}\left(\mathrm{T}_{1}\right) \\
\mathrm{CT}\end{array}$ & $\begin{array}{c}\mathrm{VPI}\left(\mathrm{T}_{1}\right) \\
\mathrm{OT}\end{array}$ & $\begin{array}{c}\mathrm{GBI}\left(\mathrm{T}_{0}\right) \\
\mathrm{CT}\end{array}$ & $\begin{array}{c}\mathrm{GBI}\left(\mathrm{T}_{0}\right) \\
\mathrm{OT}\end{array}$ & $\begin{array}{c}\mathrm{GBI}\left(\mathrm{T}_{1}\right) \\
\mathrm{CT}\end{array}$ & $\begin{array}{c}\mathrm{GBI}\left(\mathrm{T}_{1}\right) \\
\mathrm{OT}\end{array}$ \\
\hline Mean & 1.54 & 1.51 & 0.51 & 0.41 & 1.53 & 1.56 & 0.67 & 0.61 \\
\hline $\mathrm{SD}$ & 0.35 & 0.37 & 0.23 & 0.23 & 0.36 & 0.42 & 0.29 & 0.28 \\
\hline $\mathrm{Cl}$ & 1.45 & 1.42 & 0.45 & 0.35 & 1.44 & 1.45 & 0.59 & 0.54 \\
& 1.63 & 1.61 & 0.57 & 0.47 & 1.62 & 1.66 & 0.74 & 0.68 \\
\hline Median & 1.47 & 1.46 & 0.52 & 0.37 & 1.52 & 1.54 & 0.62 & 0.59 \\
\hline Min; & $1.21 ;$ & $1.20 ;$ & $0.00 ;$ & $0.05 ;$ & $0.75 ;$ & $0.62 ;$ & $0.12 ;$ & $0.15 ;$ \\
\hline Max & 3.50 & 3.87 & 1.00 & 1.55 & 2.80 & 2.90 & 1.46 & 1.40 \\
\hline IR & 0.30 & 0.23 & 0.32 & 0.24 & 0.46 & 0.44 & 0.46 & 0.40 \\
\hline
\end{tabular}

$\mathrm{CT}=$ Conventional toothbrush; $\mathrm{OT}=$ Orthodontic toothbrush; $\mathrm{SD}=$ Standard deviation; $\mathrm{Cl}=$ Confidence interval; $I R=$ Interquartile range.

Table 2 - Intergroup comparison of VPI and GBI baseline and follow-up data.

\begin{tabular}{|c|c|c|c|c|c|c|c|c|}
\hline & $\begin{array}{c}\text { VPI }\left(T_{0}\right) \\
C T\end{array}$ & $\begin{array}{c}\text { VPI }\left(T_{0}\right) \\
\text { OT }\end{array}$ & $\begin{array}{c}\mathrm{VPI}\left(\mathrm{T}_{1}\right) \\
\mathrm{CT}\end{array}$ & $\begin{array}{c}\operatorname{VPI}\left(T_{1}\right) \\
\text { OT }\end{array}$ & $\begin{array}{c}G B I\left(T_{0}\right) \\
C T\end{array}$ & $\begin{array}{c}\text { GBI }\left(\mathrm{T}_{0}\right) \\
\text { OT }\end{array}$ & $\begin{array}{c}\mathrm{GBI}\left(\mathrm{T}_{1}\right) \\
\mathrm{CT}\end{array}$ & $\begin{array}{c}\mathrm{GBI}\left(\mathrm{T}_{1}\right) \\
\text { OT }\end{array}$ \\
\hline Mean & - & - & - & - & 1.53 & 1.56 & 0.67 & 0.61 \\
\hline Median & 1.47 & 1.46 & 0.52 & 0.37 & - & - & - & - \\
\hline $\begin{array}{c}\text { Difference } \\
\text { (CT-OT) }\end{array}$ & \multicolumn{2}{|c|}{0.01} & \multicolumn{2}{|c|}{0.15} & \multicolumn{2}{|c|}{0.03} & \multicolumn{2}{|c|}{0.06} \\
\hline P Value & \multicolumn{2}{|c|}{0.678} & \multicolumn{2}{|c|}{$0.002^{*}$} & \multicolumn{2}{|c|}{0.689} & \multicolumn{2}{|c|}{0.275} \\
\hline
\end{tabular}

$\mathrm{CT}=$ Conventional toothbrush; OT $=$ Orthodontic toothbrush; *Statistically significant, $\mathrm{P} \leq 0.05$.

would become stained, which in turn could have influenced the adherence to the study. Moreover, in the present study there was no intention to achieve complete elimination of the biofilm, something that could have hindered the detection of statistically significant differences between the two methods at follow-up. The same rationale was used elsewhere ${ }^{3}$ to investigate the efficacy of manual and electric toothbrushes.

In addition to the VPI, Löe and Silness's GBI ${ }^{9}$ was considered. Because the presence and ease of gingival bleeding is dependent on the intensity of inflammation, the GBI appears to be quite suitable to study possible correlations between clinical and histological conditions.

To ascertain that the examiner was fully calibrated to apply both indexes, two experienced periodontic professors (C.F.S. and F.R.G.S.) participated in a series of training sessions to evaluate the examiner's accuracy and consistency before initiating the trial.

Although the patients in the present study were not shown product packaging nor did they receive additional information regarding the toothbrushes being tested, they could easily see the differences between the two types of toothbrushes. With this in mind, it is not possible to ascertain if the statistically significant difference between the VPI values at $T_{1}$ was a true effect of the orthodontic toothbrush or a placebo effect. Whichever the case, a difference of only 0.15 (Table 2) for VPI does not seem to be relevant from the clinical standpoint. This lack of substantial difference between conventional and orthodontic toothbrushes has also been mentioned in previous studies. ${ }^{11-13}$

Regardless of the type of toothbrush, there was a considerable reduction in both the VPI and GBI at the end of the trial for all groups, possibly indicating benefits of the overall oral hygiene program (including audio-visual resources). ${ }^{16}$ Alternatively, such results might reflect the one-on-one contact between study participants and the researcher and the quality of the rapport established between them. This has been described in the literature as the Hawthorne effect. $^{17}$

Of the four studies ${ }^{7,11-13}$ systematically obtained 
from the "PubMed" database up to June 17, 2010, only two $\mathrm{w}^{7,12}$ actually compared manual orthodontic and conventional toothbrushes. Among them, only one $^{7}$ observed greater efficacy of orthodontic toothbrushes, and this difference was limited to the anterior region of the mouth, and was considered by the authors of the original study to be of small clinical significance. In addition, another ${ }^{12}$ of these studies, using a similar experimental design and a slightly larger sample size (31 patients), was unable to verify

\section{References}

1. Goh HH. Interspace/interdental brushes for oral hygiene in orthodontic patients with fixed appliances. Cochrane Database Syst Rev. 2007 Jul 18;(3):CD005410.

2. Clerehugh V, Williams P, Shaw WC, Worthington HV, Warren $P$. A practice-based randomised controlled trial of the efficacy of an electric and a manual toothbrush on gingival health in patients with fixed orthodontic appliances. J Dent. 1998 Nov;26(8):633-9.

3. Costa MR, da Silva VC, Miqui MN, Colombo AP, Cirelli JA. Effects of ultrasonic, electric, and manual toothbrushes on subgingival plaque composition in orthodontically banded molars. Am J Orthod Dentofacial Orthop. 2010 Feb;137(2):22935.

4. Schatzle M, Sener B, Schmidlin PR, Imfeld T, Attin T. In vitro tooth cleaning efficacy of electric toothbrushes around brackets. Eur J Orthod. 2010 Oct;32(5):481-9.

5. Parizi MT, Mohammadi TM, Afshar SK, Hajizamani A, Tayebi M. Efficacy of an electric toothbrush on plaque control compared to two manual toothbrushes. Int Dent J. 2011 Jun;61(3):131-5.

6. Costa MR, Silva VC, Miqui MN, Sakima T, Spolidorio DM, Cirelli JA. Efficacy of ultrasonic, electric and manual toothbrushes in patients with fixed orthodontic appliances. Angle Orthod. 2007 Mar;77(2):361-6.

7. Williams P, Fenwick A, Schou L, Adams W. A clinical trial of an orthodontic toothbrush. Eur J Orthod. 1987 Nov;9(4):295304.

8. Silness J, Löe H. Periodontal Disease in Pregnancy. Ii. Correlation between Oral Hygiene and Periodontal Condtion. Acta Odontol Scand. 1964 Feb;22(1):121-35.

9. Löe H, Silness J. Periodontal Disease in Pregnancy. I. Prevalence and severity. Acta Odontol Scand. 1963 Dec;21(6):533-51. the alleged superiority of orthodontic toothbrushes.

The results of the present study also do not provide evidence in favor of the use of manual orthodontic toothbrushes for patients with fixed orthodontic appliances in the permanent dentition. At present, therefore, it would seem reasonable that patients together with their dentists should select a manual toothbrush based on comfort and likelihood of use rather than claims of greater efficacy.

10. Silvestrini Biavati A, Gastaldo L, Dessi M, Silvestrini Biavati F, Migliorati M. Manual orthodontic vs. oscillating-rotating electric toothbrush in orthodontic patients: a randomised clinical trial. Eur J Paediatr Dent. 2010 Dec;11(4):200-2.

11. Thienpont V, Dermaut LR, Van Maele G. Comparative study of 2 electric and 2 manual toothbrushes in patients with fixed orthodontic appliances. Am J Orthod Dentofacial Orthop. 2001 Oct;120(4):353-60.

12. Rafe Z, Vardimon A, Ashkenazi M. Comparative study of 3 types of toothbrushes in patients with fixed orthodontic appliances. Am J Orthod Dentofacial Orthop. 2006 Jul;130(1):925.

13. Laher A, Kroon J, Booyens SJ. Effectiveness of four manual toothbrushes in a cohort of patients undergoing fixed orthodontic treatment in an academic training hospital. SADJ. $2003 \mathrm{Jul} ; 58(6): 231,234-7$.

14. Quigley GA, Hein JW. Comparative cleansing efficiency of manual and power brushing. J Am Dent Assoc. 1962 Jul(1);65:26-9.

15. Turesky S, Gilmore ND, Glickman I. Reduced plaque formation by the chloromethyl analogue of victamine C. J Periodontol. 1970 Jan;41(1):41-3.

16. Alsada LH, Sigal MJ, Limeback H, Fiege J, Kulkarni GV. Development and testing of an audio-visual aid for improving infant oral health through primary caregiver education. J Can Dent Assoc. 2005 Apr;71(4):241, 241a-h.

17. Feil PH, Grauer JS, Gadbury-Amyot CC, Kula K, McCunniff MD. Intentional use of the Hawthorne effect to improve oral hygiene compliance in orthodontic patients. J Dent Educ. 2002 Oct;66(10):1129-35. 